\title{
Reliability Implementation and Cost Trade-off of Product during the Development Process
}

\author{
Wei Wang ${ }^{1}$, Yuan Li ${ }^{1}$, Qi Zhang ${ }^{1}$, Weijia Feng ${ }^{1}$, Huichao Liu ${ }^{1}$, Pengyan Liu ${ }^{1}$ \\ ${ }^{1}$ School of Reliability and Systems Engineering, Beihang University, Beijing, China
}

\begin{abstract}
Modern product needs to meet the reliability requirements during the development process. The reliability in this paper refers to an integral view of a product's reliability, maintainability, supportability, testability, safety and environmental adaptability. However, during the product development process, the two problems are how to evaluate the implementation and how to determine the work input costs of reliability. This paper proposes a method to evaluate the degree of reliability implementation. And it researches the schemes and targets decision-making method based on trade-off analysis. Through establishing and solving trade-off optimization model, the results can help decision makers find the optimal parameters program and cost goals.
\end{abstract}

\section{Introduction}

The developed reliability engineering integrates the separated engineering work such as reliability engineering, testability engineering and safety engineering and considers the interactions among each engineering work, making the product quality into a higher level [1]. During the development process, it is necessary to periodically evaluate the degree of completion of the reliability work aiming to ensure the product reliability meet the requirements. And the basic way of evaluation is expert review. Chen evaluates reliability of armored product by establishing evaluation index system based on RST-QFD-Bayes [2]. When choosing the development scheme for the product, determining the costs required of the reliability work and making trade-offs are critical to ensure the realization of reliability of the product more economical. The CAIV method (cost as an independent variable) proposed by the US military is a more advanced cost management method nowadays and the trade-off method part is used for reference in this paper [3-5].

\section{Reliability implementation evaluation}

The purposes of the reliability implementation evaluation are evaluating the degree of completion of the reliability work and providing the reliability implementation model for trade-off analysis.

\subsection{Reliability implementation system}

The reliability implementation system is established as Figure 1 by considering the various factors that affect the reliability implementation and the relationships among them based on the evaluation index system construction principle [6]. In order to establish the system, the reliability requirements of product and the reliability work specified in national military standards are analyzed firstly. Then the factors affecting the realization of reliability, maintainability, supportability, testability, safety and environmental adaptability are extracted and merged. Finally, the correlation among the factors is considered and the structure of reliability implementation system is determined.

\subsection{Reliability implementation evaluation based on AHP}

The analytic hierarchy process (AHP) which has the special advantage in multi-criteria evaluation is very suitable for the evaluation of this paper [6]. The evaluation process of reliability implementation based on AHP is as follows. First, establish the evaluation indexes of reliability implementation. Second, determine the weights of the first-level assessment index and the second-level assessment index. Third, calculate the evaluation value of the first-level assessment index based on the scores of the experts. Forth, calculate the evaluation value for reliability implementation degree.

\subsubsection{Evaluation indexes for reliability implementation}

The evaluation indexes are established based on the factors of reliability implementation system. The evaluation indexes are shown in Figure 2. The evaluation indexes include two layers. The first-level assessment index includes the six assessment indexes: organization, 
product and facilities, management and controlling, reliability design and analysis, reliability test and assessment, reliability attainment and improvement, which are denoted as $\mathrm{X}_{1}, \mathrm{X}_{2}, \mathrm{X}_{3}, \mathrm{X}_{4}, \mathrm{X}_{5}, \mathrm{X}_{6}$. In the second-level assessment index, organization includes the three second-level assessment indexes: staffing, agency, post, which are denoted as $\mathrm{R}_{11}, \mathrm{R}_{12}, \mathrm{R}_{13}$ respectively. Product and facilities includes the three second-level assessment indexes: facilities and environmental conditions, product allocation, product maintenance, which are denoted as $\mathrm{R}_{21}, \mathrm{R}_{22}, \mathrm{R}_{23}$ respectively. Management and controlling includes the eight secondlevel assessment indexes: plan, procedure controlling, review management, closed-loop controlling and management, information management, hierarchical management, state management, material controlling and management, which are denoted as $R_{31}, R_{32}, R_{33}, R_{34}, R_{35}$, $\mathrm{R}_{36}, \mathrm{R}_{37}, \mathrm{R}_{38}$ respectively. Reliability design and analysis includes the six second-level assessment indexes: reliability design guidelines, reliability model, reliability distribution and forecast, reliability failure and hazard analysis, design and iteration, integrated design, which are denoted as $\mathrm{R}_{41}, \mathrm{R}_{42}, \mathrm{R}_{43}, \mathrm{R}_{44}, \mathrm{R}_{45}, \mathrm{R}_{46}$ respectively. Reliability test and assessment includes the four secondlevel assessment indexes: engineering test, synthetic test, simulation, comprehensive evaluation, which are denoted as $R_{51}, R_{52}, R_{53}, R_{54}$ respectively. Reliability attainment and continuous improvement includes the three secondlevel assessment indexes: qualification, out-field performance, continuous improvement, which are denoted as $\mathrm{R}_{61}, \mathrm{R}_{62}, \mathrm{R}_{63}$ respectively.

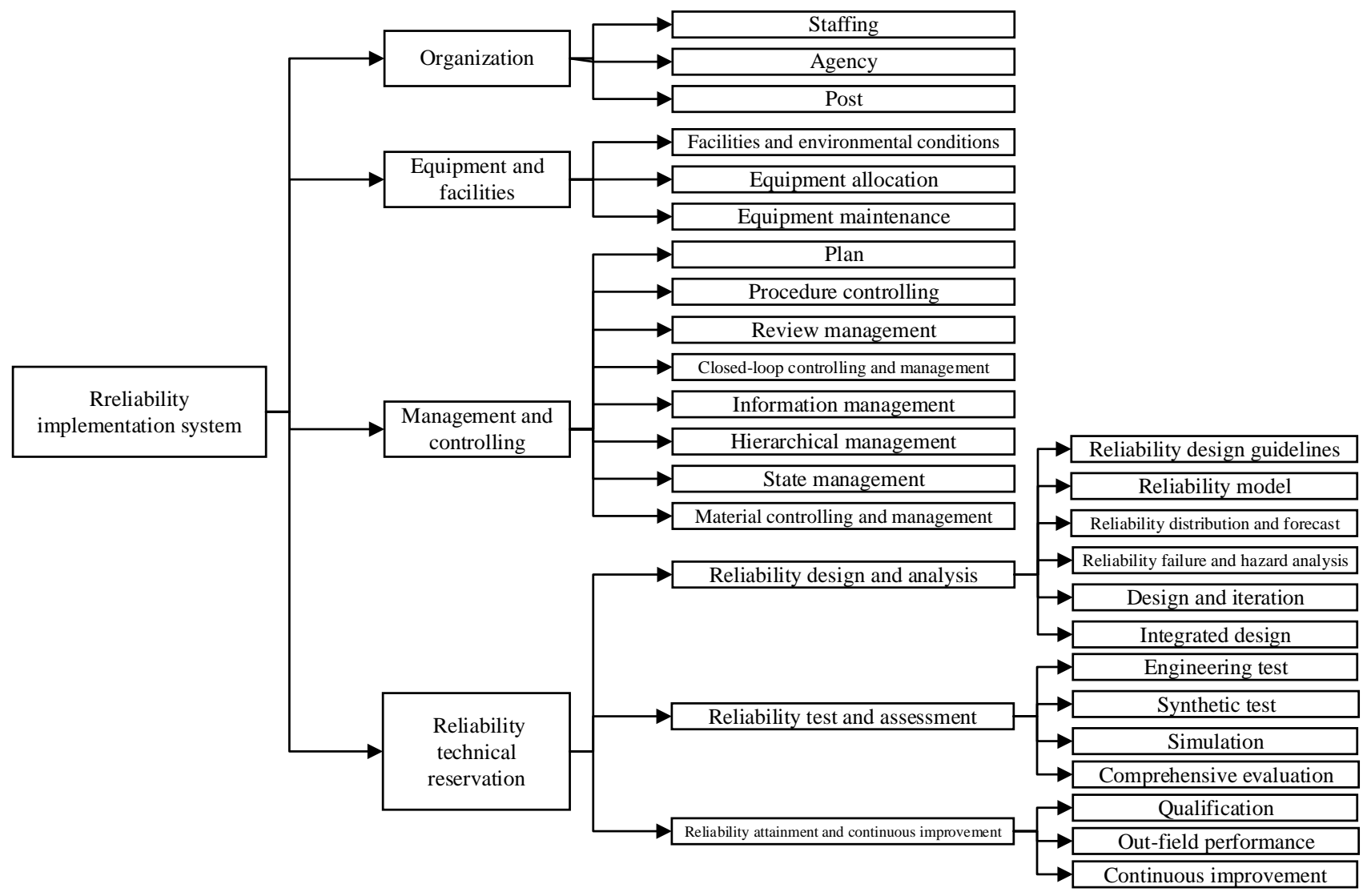

Figure 1. Reliability implementation system.

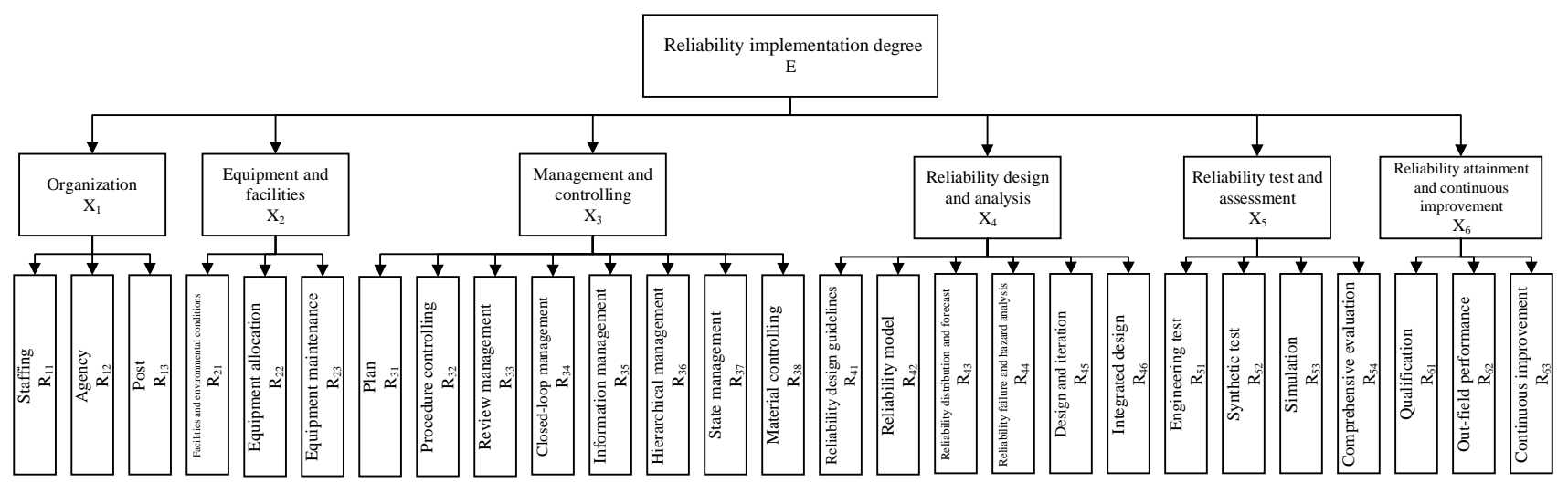

Figure 2. Evaluation indexes of reliability implementation. 


\subsubsection{Weight Calculation of the Assessment Index}

This paper uses the AHP method to establish a weight set, which classifies various related factors in a complex problem into an interrelated and orderly hierarchy. Based on certain objective judgments, the first-level and the second-level assessment indexes are compared and analyzed. In the weight calculation of the two layers of assessment indexes, some experts are invited to construct the discriminant matrix by comparing the index among each other. 1 9 are used as comparison scales among evaluation factors. By solving these discriminant matrices, it can be calculated that the six weights of the first-level assessment index for reliability implementation degree which are denoted as $\omega_{i}(i=1,2,3,4,5,6)$ and the weights of the second-level assessment index for the first-level assessment indexes which are denoted as $\omega_{i j}(i=$ $1,2,3,4,5,6 ; j=1,2 \cdots)$.

\subsubsection{Calculation of evaluation model}

The evaluation value for reliability implementation degree is calculated by values and weights of every evaluation index, which is expressed as:

$$
E=\sum_{i=1}^{6} X_{i} \omega_{i}
$$

where $\omega_{i}(i=1,2,3,4,5,6)$ is the weight of the first-level assessment index; $X_{i}(i=1,2,3,4,5,6)$ is the value of the first-level assessment index, which is expressed as:

$$
X_{i}=\sum_{\mathrm{j}=1}^{\mathrm{n}_{i}} R_{i j} \omega_{\mathrm{ij}}
$$

where $\omega_{i j}(i=1,2,3,4,5,6 ; j=1,2 \cdots)$ is the weight of the second-level assessment index; $R_{i j}(i=$ $1,2,3,4,5,6 ; j=1,2 \cdots)$ is the value of the second-level assessment index, which is scored by experts during the evaluation.

In the actual evaluation, the second-level assessment index need to be decomposed into the third-level or even fourth-level index to evaluate the values. In the subsequent related studies, we will give more details. The value of the second-level assessment index will reflect the completion degree in the form of percentage, which means from 0 to $100 \%$.

\subsubsection{Result analysis}

The reliability implementation degree can be obtained through calculating the weights and evaluating. the values of the indexes. The reliability implementation degree is a value between 0 and $100 \%$ can be rated five levels. The corresponding level and significance of each value are shown in Table 1.

Table 1. Reliability implementation degree and level correspondence table.

\begin{tabular}{ccl}
\hline E & Level & \multicolumn{1}{c}{ Significance } \\
\hline$[0,30 \%)$ & I & $\begin{array}{l}\text { Reliability work is basically not } \\
\text { implemented and the product } \\
\text { cannot meet the requirements. } \\
\text { Reliability work centered on }\end{array}$ \\
\hline $30 \%, 55 \%)$ & II & R
\end{tabular}

typical projects are implemented and the product cannot meet the requirements. Most reliability work are [55\%,75\%] III implemented and the product partially meet the requirements. All reliability work is $(75 \%, 90 \%]$ IV implemented and the product basically meet the requirements. Reliability work is fully $(90 \%, 100 \%] \quad \mathrm{V}$ implemented and the product meet the requirements.

\section{Trade-off analysis between reliability implementation and cost}

The process of reliability implementation and cost tradeoff analysis can be described as follows: according to certain evaluation criteria, the evaluation value of each alternative scheme can be calculated by establishing and solving trade-off model, then the alternative scheme with the highest evaluation value can be selected as the optimal decision. A more detailed process is shown in Figure 3.

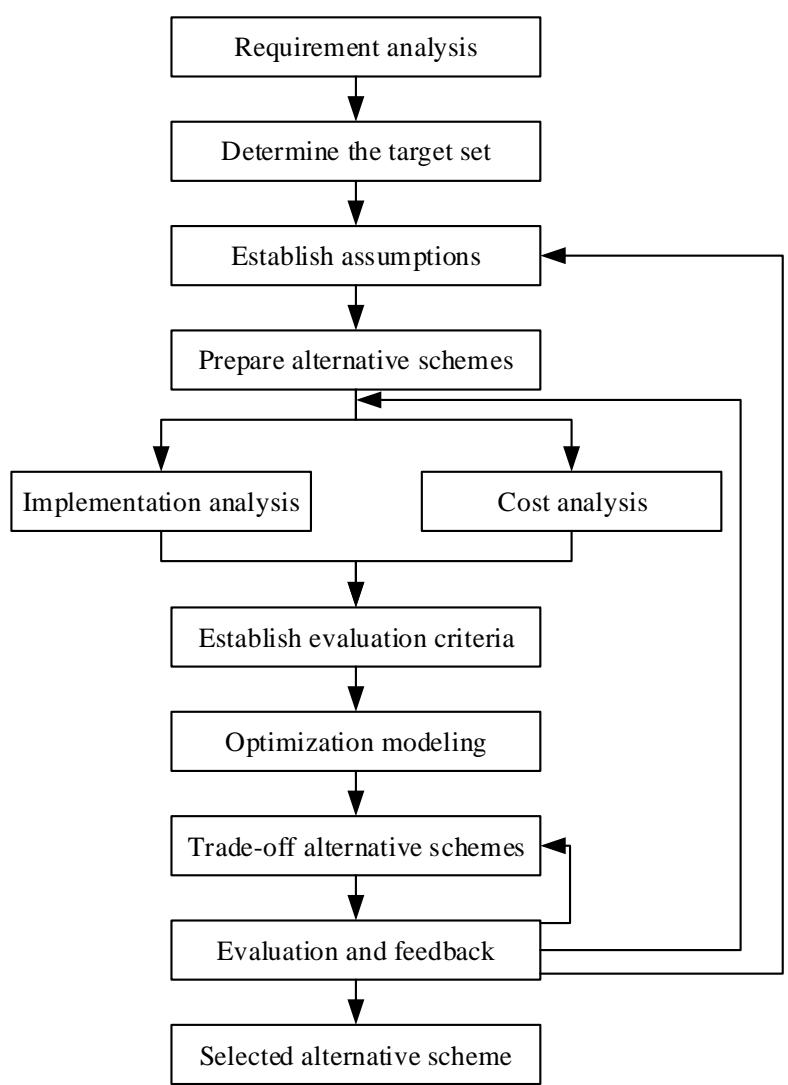

Figure 3. Process of reliability implementation and cost tradeoff analysis.

In order to establish a link between reliability implementation and cost for trade-off, the first-level assessment index of reliability implementation evaluation is used as a decision variable to participate in trade-offs as parameters that connect reliability implementation with cost. The optimal solution consisting of the decision 
variable can be obtained by establishing and solving the trade-off model.

\subsection{Reliability implementation model and cost model}

Since equation (1) can measure the degree of reliability implementation, it can be used as the reliability implementation model for trade-off. In this section, it is written as equation (3):

$$
\mathrm{E}=f\left(X_{1}, X_{2}, X_{3}, X_{4}, X_{5}, X_{6}\right)
$$

The cost model can be established by multiple linear or nonlinear regression using the evaluation results of reliability implementation and costs of some similar product in the past [7]. The cost model is as follows:

$$
\mathrm{C}=g\left(X_{1}, X_{2}, X_{3}, X_{4}, X_{5}, X_{6}\right)
$$

where $\mathrm{C}$ is the cost of the reliability work.

\subsection{Trade-off analysis}

Not only the indexes of reliability implementation model have a certain range of values, but also the cost of reliability work must be subject to certain constraints. Therefore, when making trade-off analysis, it is necessary to specify the value range, which is also called the tradeoff space which is shown in Figure 4. The trade-off space can be described as a multi-dimensional closed space. It represents a feasible set of solutions. In this paper, the trade-off space refers to the maximum value range of the first-level assessment index of evaluation and the maximum cost limit.

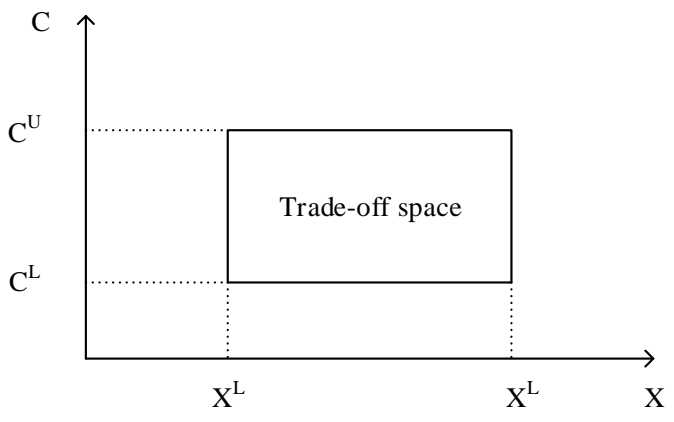

Figure 4. Trade-off space of cost and index of reliability implementation.

Goal of trade-off analysis is to obtain the best decision of the reliability implementation degree and cost, which means minimum cost and maximum reliability implementation degree are both the goals. This goal could be expressed as a function composed of $\mathrm{E}$ and $\mathrm{C}$, as shown in equation (5):

$$
\mathrm{K}=\mathrm{F}(E, C)
$$

where $\mathrm{K}$ is the evaluation value of the alternative schemes; $\mathrm{F}$ is the evaluation criteria.

Evaluation criteria means the trade-off principles of reliability implementation and cost. The most commonly used evaluation criteria are maximizing the reliability implementation, minimizing the cost of reliability work, maximizing the reliability implementation cost ratio. The evaluation criteria are chosen based on the resources, economic, political and so on. In this paper, a trade-off model is given as equation (6).

$$
\begin{gathered}
\max \mathrm{K}=\mathrm{F}(E, C) \\
\text { s.t. } \mathrm{E}=f\left(X_{1}, X_{2}, X_{3}, X_{4}, X_{5}, X_{6}\right) \geq E_{0} \\
\mathrm{C}=g\left(X_{1}, X_{2}, X_{3}, X_{4}, X_{5}, X_{6}\right) \leq C_{0} \\
X_{i}^{L} \leq X_{i} \leq X_{i}^{U}(i=1,2,3,4,5,6)
\end{gathered}
$$

where $X_{i}^{L}$ and $X_{i}^{U}$ means the lower and upper limits of the decision variable $X_{i}$.

\section{Conclusion}

This paper proposes a reliability implementation system based on the research of the reliability work of product during the development process. This system includes the factors that affect the reliability implementation and is used as the evaluation object to establish an AHPbased evaluation method that can be applied to the periodic review of product development process and to guide the reliability work. In addition, the paper also proposes a method of trade-off the reliability implementation degree with the cost of reliability work, which can guide the input and implementation of reliability and increase the economic efficiency of reliability work during the development process. One of the great advantages of the evaluation method and trade-off method is that reliability management problem can be solved well in the absence of actual understanding and data in the development process.

Further study will consider the constraints of risk control to perfect the reliability implementation model and the cost model and to make the solution more instructive.

\section{References}

1. Zhao Qing-li, "An Analysis and Discussion on the Implementation of Common Quality Characteristic Standards," Electronic Quality. 44-48(2015)

2. Chen Chunliang and Liu Yan, "Research on Establishing Evaluation Index System of New Type Armored Product Common Quality Characters Based on RST-QFD-Bayes," Computer Measurement \& Control. 2438-2442(2015)

3. J. Brady, "Systems engineering and cost as an independent variable," Systems Engineering, vol. 4, no. 4, pp. 233-241(2001)

4. Q.S. Guo and L. Zhang, "Research summary of weapons and product systems effectiveness evaluation methods," Computer Simulation, vol. 30, no. 8, pp. 1-4(2013)

5. Karen Tyson, John Hiller, David Hunter. Assessing Cost-Reduction Initiatives in a Changing Defense Acquisition Environment-A Handbook. Alexandria, Virginia: Institute for Defense Analysis(1998)

6. L. Breiman, "Hinging hyperplanes for regression, classification, and function approximation," IEEE Transactions on Information Theory, vol. 39, no. 3, pp. 999-1013(1993) 
7. T.A. Stukel, "Generalized logistic models," 402, pp. 426-431(1988) Mathematical \& Computer Modelling, vol. 83, no. 